\title{
La carencia de valores, una de las de las causas de la deficiente recaudación fiscal en México
}

\section{The lack of securities, one of the causes of the deficient tax collection in Mexico}

HERRERA-CHAIRES, María del Carmen $\dagger^{*}$, GAMBOA-CERDA, Silvia, MEDELLIN-RAMIREZ, Juan Emilio y VILLEGAS-FLORES, Ignacio

Universidad Autónoma de San Luis Potosí, Facultad de Contaduría y Administración. Alvaro Obregon 64, Centro, 78300 San Luis, S.L.P.

ID $1^{\text {er }}$ Autor: Maria del Carmen, Herrera-Chaires / ORC ID: 0000-0003-2666-2152, Researcher ID Thomson: X-79632018, CVU CONACYT ID: 380993

ID $1^{\text {er }}$ Coautor: Silvia, Gamboa-Cerda / ORC ID: 0000-0002-2555-3918, Researcher ID Thomson: X-8584-2018, CVU CONACYT ID: 323123

ID $2^{\text {do }}$ Coautor: Juan Emilio, Medellin-Ramirez / ORC ID: 0000-0003-4634-9913, Researcher ID Thomson: X-87912018, CVU CONACYT ID: 954329

ID $3^{\text {er }}$ Coautor: Ignacio, Villegas-Flores / ORC ID: 0000-0003-4672-2044, Researcher ID Thomson: X-4085-2019, CVU CONACYT ID: 6429074

DOI: $10.35429 / J L A .2019 .8 .3 .32 .43$

\section{Resumen}

En el presente trabajo se inspira a partir de explicar en lo posible con otra óptica el problema fiscal de México. Abordándose temas como la cultura cívica y las costumbres de los mexicanos respecto al optimismo y pesimismo colectivo y cómo influye en el desarrollo de nuevas ideas o cambio de hábitos sociales, todo para ofrecer un primer acercamiento al problema fiscal de México. De hecho, en México es muy difícil definir los niveles reales de evasión y se inclina a analizar el problema fiscal desde la ética y los valores, se estudia como este tema que ha sido un tanto descuidado en la actualidad y cómo influye fuertemente sobre el comportamiento de las personas, haciendo las observaciones desde cómo afecta en el ámbito de los impuestos. Analiza los valores que practica la juventud y los contrasta con las generaciones mayores, abarcando como opinan los ciudadanos a los temas que rodean la problemática fiscal. Se presentan los resultados de la investigación en torno a los elementos que influyen en la formación de las nuevas generaciones, y se analiza si estos elementos resultan positivos o perjudícales.

Valores, Recaudación fiscal, Ética

\begin{abstract}
This work is inspired by explaining as far as possible with another perspective the fiscal problem of Mexico. Addressing issues such as civic culture and customs of Mexicans regarding optimism and collective pessimism and how it influences the development of new ideas or changing social habits, all to offer a first approach to the fiscal problem of Mexico. In fact, in Mexico it is very difficult to define the real levels of evasion and it is inclined to analyze the fiscal problem from ethics and values, it is studied as this issue that has been somewhat neglected today and how it strongly influences the behavior of people, making observations from how it affects taxation. It analyzes the values practiced by youth and contrasts them with older generations, covering as citizens think about the issues surrounding fiscal issues. The results of the research are presented around the elements that influence the formation of the new generations, and it is analyzed whether these elements are positive or harmful.
\end{abstract}

Securities, Tax collection, Ethics

Citación: HERRERA-CHAIRES, María del Carmen, GAMBOA-CERDA, Silvia, MEDELLIN-RAMIREZ, Juan Emilio y VILLEGAS-FLORES, Ignacio. La carencia de valores, una de las de las causas de la deficiente recaudación fiscal en México. Revista de Aplicaciones del Derecho. 2019. 3-8: 32-43.

\footnotetext{
*Correspondencia al Autor (Correo Electrónico: chchaires@gmail.com)

$\dagger$ Investigador contribuyendo como primer autor.
} 


\section{Antecedentes}

En cierto sentido la historia de México, como la de cada mexicano, consiste en una lucha entre las formas y fórmulas en que se pretende encerrar a nuestro ser y las explosiones con que nuestra espontaneidad se venga. Pocas veces la Forma ha sido una creación original, un equilibrio alcanzado no a expensas sino gracias a la expresión de nuestros instintos y quereres. Nuestras formas jurídicas y morales, por el contrario, mutilan con frecuencia a nuestro ser, nos impiden expresarnos y niegan satisfacción a nuestros apetitos vitales (Paz, 1981, págs. 1112). El escritor Octavio Paz busca, a través de la narrativa anterior, establecer una imagen con la que identifica y califica a los mexicanos de los años cincuenta; pese a que encajona en ella a la totalidad de los habitantes de México de esa época en una sola descripción, logró revelar una serie de características que le son comunes a la mayoría de los mexicanos.

La identidad de los mexicanos es compleja y multifacética, desprendida de una herencia de conquista y mestizaje, de una historia de traiciones, guerra e inestabilidad, del pasado indígena que algunas veces es ensalzado y otras veces es menospreciado, de un proceso de independencia que sirvió a los intereses de los poderosos de la época, de una revolución que se llevó a cabo con un sinfín de motivaciones sin homologar, de un México contemporáneo impregnado con la mancha de corrupción, con funcionarios públicos abusivos que solo han buscado, por años, vivir del erario público. Esta historia común a todos los nacidos y nacionalizados en este territorio han servido de contexto para enfrentar y soportar sus problemas sociales, políticos y económicos, problemas como la inseguridad, la injusticia, las diferencias sociales, las carencias del sistema de seguridad social y muchos otros abusos que son el pan de cada día.

En contraparte, el gobierno habla de que no tiene el presupuesto suficiente para atender las necesidades de la población de manera integral y así se desata un estira y afloja interminable en el ámbito fiscal de un pueblo insatisfecho y un gobierno que ha sido incapaz de realizar su función; en tal situación todo gira en torno a que, por un lado la población siente impotencia ante el abuso por parte del gobierno en cuanto al mal manejo de los recursos y la carga fiscal lo que genera un ánimo poco cooperativo de los ciudadanos con la autoridad.
Por el otro, lado el gobierno incapaz de resolver dicho problema, se avoca a administrar la "austeridad" para sus gobernados pero a pesar de ello sigue habiendo el despilfarro en beneficio de los burócratas (bonos, premios y prestaciones especiales) y en algunos casos en su beneficio individual personal.

Es este panorama el que motiva el presente estudio y a desentrañar si es posible mirar con otra óptica el problema fiscal de México. De hecho en México es muy difícil definir los niveles reales de evasión. ¿Por qué? Por ser un país con un alto índice de corrupción donde los datos "oficiales" no son 100 por ciento confiables, donde no cuesta trabajo imaginar que los datos son "cuchareados". Es cierto que los esfuerzos y las medidas para controlar a los contribuyentes se han llevado a cabo y que se ha trabajado en eliminar las lagunas jurídicas que favorecían a los que eluden sus obligaciones fiscales, sin embargo, la situación fiscal que se percibe en el país no coincide con algunos de los datos que presenta la Secretaría de Hacienda y Crédito Público al respecto, sobre todo al contrastar con lo que presentan otros estudios en la materia por parte de instituciones $\mathrm{u}$ organismos internacionales como la CEPAL (Comisión Económica para América Latina y el Caribe) quien realiza un estudio en el que incluye a México respecto a la eficiencia recaudatoria y lo califica de pobre (Aristegui noticias, 2012).

\section{Planteamiento del problema}

La reforma hacendaria que entró en vigor a partir de 2014 vuelve a exponer la complejidad del sistema tributario mexicano, el cual queda por demás claro que es ineficiente. Se siguen buscando formas de recaudar más recursos pero, debido a la repetición de medidas aplicadas con sus distintas variantes, siempre salen afectados los mismos empresarios dueños de pequeñas y medianas empresas, los mismos asalariados y los mismos profesionistas independientes, también conocidos como freelancers. En general, gente que produce, que se compromete y que aporta beneficios a México por la vía del trabajo digno y honrado.

HERRERA-CHAIRES, María del Carmen, GAMBOA-CERDA, Silvia, MEDELLIN-RAMIREZ, Juan Emilio y VILLEGAS-FLORES, Ignacio. La carencia de valores, una de las de las causas de la deficiente recaudación fiscal en México. Revista de Aplicaciones del Derecho. 2019 
En esta ocasión, el manejo mediático se llevó a cabo con una destreza tal, que la mayoría discutía temas irrelevantes como: si "el chicle es un alimento o no para poder ser gravado con IVA", "la diferencia entre goma de mascar y chicle" o "si las mascotas son un lujo o no para ser objeto de tributo", "si las personas que viven solas y gozan de la compañía de mascotas se ven afectadas" o "si el aumento en el costo de bebidas con alto contenido calórico afectaría a los más pobres". Mientras tanto, se dejaron de lado los debates verdaderamente importantes, no hubo mucha difusión de información, ni se expusieron los debidos análisis de los temas de mayor relevancia como: la fiscalización vía tarjetas de crédito, la desaparición del régimen del pequeño contribuyente, el pago del 10 por ciento para dividendos (existiendo una doble tributación en este caso), el impacto en materia de comercio exterior por concepto de IVA del 16 por ciento en la frontera y las modificaciones en materia de importaciones temporales $\mathrm{y}$, por último, nadie habló tampoco de cómo garantizaría la autoridad la protección de los datos personales ya que con muchas de las medidas que se implementarían, la autoridad conocerá cada paso que los contribuyentes den y se debería garantizar que esta intima información no pueda caer en malas manos. En años pasados se ha dicho que una verdadera y justa solución para el problema fiscal en México es aumentar la base de contribuyentes, lo cual esencialmente es correcto; pero no es la única acción que se debe realizar. No hay que olvidar que los recursos captados por pago de contribuciones, impuestos y derechos tienen una finalidad específica, que es que el Estado opere y realice acciones que beneficien a la sociedad que los paga; sin embargo, eso no sucede siempre. Los recursos captados, pocos o muchos, en bastantes ocasiones se emplean tomando como base el interés personal de los funcionarios públicos en turno sobre el bien de la colectividad, acciones de corrupción y peculado aparecen todos los días en las noticias del país ¿Cómo afectan estas acciones la postura de los contribuyentes?

Es entonces, como nace el supuesto de que la mitad del problema fiscal de México radica para la administración pública, por la falta de actuación ética y la ausencia de honradez, integridad y diligencia; y la otra mitad del problema son los evasores de impuestos.
Este representa un serio problema y forma parte del reto a vencer en materia fiscal.

Todo esto se convierte en una maraña de engaños donde existen, por ejemplo, casos de funcionarios que incluyen en la nómina a familiares y amigos y los evasores de impuestos se cuestionan “¿Para qué pago si de todos modos se lo van a robar?

Ante todo ello, ¿Cuál es la consecuencia de este fenómeno donde nadie se responsabiliza y todos se justifican con los actos de la contraparte?

Es por ello, que el planteamiento de la presente investigación hace un intento por alejarse de lo tradicional, desea ofrecer pistas que coadyuven en resolver de raíz el problema fiscal y no solo proponiendo modificar la legislación, modificar tasas, otorgar incentivos para el pago, implementar sistemas electrónicos y demás medidas que ya se han tomado y discutido durante décadas.

El estudio se enfocó en analizar el problema fiscal en México desde la óptica de los valores, la ética, la autorregulación de los contribuyentes y de los políticos y en favor de la dignidad humana alentar a hacer honestamente lo que a cada uno le corresponde por la sociedad.

Es necesario que enfoques del tipo como el que se plantea en esta investigación, vean la luz porque, como dijo en alguna ocasión Albert Einstein (año), la locura es: seguir haciendo lo mismo y esperar resultados diferentes. Si se busca resolver el problema fiscal en México, resulta necesario generar ideas nuevas, propuestas diferentes y enfoques fuera de lo convencional que arrojen nuevas posibilidades y alternativas para mejorar la situación recaudatoria del país.

\section{Preguntas de investigación}

Del interés por realizar la presente investigación se derivaron de una serie de interrogantes que marcaron la línea de investigación entre las cuales se pueden mencionar las siguientes:

- ¿Qué influye para la formación ética y de los valores en los mexicanos? 
- ¿Existen diferencias en los valores que viven las nuevas generaciones en comparación con las anteriores?

- ¿Cómo afecta el peculado y la inadecuada administración de los recursos públicos los sentimientos de los contribuyentes?

\section{Objetivo general}

- Identificar la relación que existe entre la forma en que las personas viven los valores morales y la tendencia que muestran ante el cumplimiento de las obligaciones fiscales, a fin de sentar precedentes que permitan abordar el problema de los impuestos desde la óptica del comportamiento ético de los ciudadanos.

\section{Objetivos específicos}

- Identificar los valores presentes en los contribuyentes cumplidos y en los evasores.

- Revisar las variaciones que se presentan en los valores que viven los contribuyentes según la generación a la que pertenezcan.

Esta investigación se realizó en la ciudad de San Luis Potosí, para lo cual se aplicó un instrumento de investigación consistente en una encuesta de veinticinco reactivos. Se encuestó de manera aleatoria a trabajadores de todos los rangos jerárquicos de las empresas localizadas en el parque industrial Tres Naciones, tales como: Cummins Filtración, Waukesha, Tiger Drylac y Fagor.

Del mismo modo el instrumento de investigación antes referido se aplicó a diversos alumnos del posgrado de la Facultad de Contaduría y Administración de la UASLP inscritos en la maestría en administración con énfasis en negocios, impuestos y gestión pública.

El trabajo se completó con una revisión documental de diversos estudios realizados a nivel nacional tal fue el caso de la Encuesta Nacional de Valores en Juventud, y algunos estudios publicados en la revista Nexos que abarcan habitantes del Distrito Federal y área metropolitana con el propósito de elevar el alcance del mismo.
Es importante estudiar el problema fiscal en México que involucra tanto la evasión, la elusión y muchas veces el "robo" de los recursos públicos desde una óptica menos rígida, en comparación con la óptica de otros trabajos que los hacen desde las leyes, la contabilidad y la administración pública, a fin de darle una oportunidad al civismo, a la filosofía y a la psicología, que aunque pareciera que son ciencia ajenas y que no tienen las bases teóricas para abordar el tema, lo cierto es que quienes administran los recursos y quienes abastecen el presupuesto de egresos son personas y la ética y los valores les son inherentes.

La contabilidad no trata de personas, las leyes disponen lineamientos para que la gente actúe y si no se siguen dichas reglas, entonces determina los castigos que se deberán aplicar en tal caso, pero se olvida de las motivaciones reales que llevan a los individuos a realizar diferentes actos, motivos que pueden ir desde lo económico, lo social e incluso lo emotivo que puede haber en torno a cada situación; así mismo, el actuar del ciudadano no es, en todos los casos, de forma autómata, por el contrario, se cuestiona el porqué de las cosas y al hacerlo se encuentran los "recovecos" para llevar a cabo su voluntad en beneficio de sus propios intereses, aun cuando ello contravenga a la ley aplicable a cada caso.

Es también de importancia, considerar el enfoque que implica que los mexicanos actúan a través de sus valores, costumbres y herencia cultural del mestizaje porque esto es el ADN social de los mexicanos, las cosas que comparten que les da cierta identidad para actuar de manera similar, lo cual es el núcleo de la investigación. No se puede resolver el problema fiscal de un país como México haciendo análisis solamente desde lo material sin contemplar el elemento humano y sus componentes.

\section{Marco Teórico}

Como lo expone Savater, la ética es el estudio de lo que es bueno o lo que es malo para el hombre, atinadamente el autor realiza el análisis sin influencia o implicaciones religiosas o subjetivas o como Immanuel Kant (2008) explica, lo importante es determinar si los actos del ser humano llevan a vivir mejor al hombre o no.

HERRERA-CHAIRES, María del Carmen, GAMBOA-CERDA, Silvia, MEDELLIN-RAMIREZ, Juan Emilio y VILLEGAS-FLORES, Ignacio. La carencia de valores, una de las de las causas de la deficiente recaudación fiscal en México. Revista de Aplicaciones del Derecho. 2019 
Se debe considerar que, no siempre un acto jurídico es necesariamente ético, por ejemplo, quien paga una deuda puede hacerlo por dos motivos, por la honorabilidad de cumplir con una obligación adquirida o por miedo al castigo, siendo que la segunda es en apego a las normas jurídicas y no es precisamente porqué se trate de un acto ético en esencia.

La ética también es denominada la doctrina de la virtud, porque quien sigue las leyes coactivas no por ende es virtuoso sino más bien obediente; entonces la virtud no atañe exactamente a la bondad moral, sino que tiene un sentido de la fortaleza del ánimo en el dominio de uno mismo el cual está relacionado a la intencionalidad moral de los actos a realizar.

Kant explica muchos aspectos de relevancia, aplicables tanto para los contribuyentes que faltan a la ley con la evasión y la elusión, como para los políticos que cometen defraudación. Por ejemplo, si en ambos casos, aplicaran los principios de la escuela cínica, habría menos "tentaciones" de defraudar al fisco, si el servidor público fuera desprendido y poco ostentoso y encontrara satisfacción en la virtud de realizar su trabajo con dignidad junto con el fundamento de servir al pueblo, o más aun, simplemente por ganar el dinero que él y su familia requieren para satisfacer sus necesidades principales, no sería tentado a corromperse y se respetaría a sí mismo, sería más feliz y más digno y así el supremo bien es alcanzado.

En el desarrollo de esta investigación se presentó la dificultad de recabar fuentes legítimas que permitan exponer una definición clara y universal de los valores éticos. Por lo que se tomó la decisión de remitirse en primera instancia a algunas de las definiciones de la palabra "valor" que ofrece la Real Academia de la Lengua Española (2014).

Si se busca proponer nuevas formas de ver el problema fiscal en México y con ayuda del presente estudio encontrar que hay una relación entre los valores de los ciudadanos, la evasión de impuestos y el hecho de que las nuevas generaciones han cambiado su forma de vivir los valores, se debe tomar con calma el asunto, evaluar, profundizar, planear estrategias y darle continuidad a las acciones.
Se ha visto en reiteradas ocasiones que cuando se quiere hacer conciencia social o informar a la gente respecto a algo, se lanzan spots en radio y televisión, entre otros medios, incluso se forman grupos que se acercan a la población para difundir el mensaje y todo pinta muy bien al inicio, sin embargo, después del relumbrón de estas acciones, se deja de lado el esfuerzo y se pierde la continuidad, esta es la razón por la cual, las campañas positivas de difusión no logran el efecto esperado en la población.

Entonces ¿Qué pasa con los valores en México? En el país no se ha abordado lo suficiente el rol que juegan los valores en la conformación de las instituciones y la sociedad. De seguirse viendo como elementos separados, esta nación seguirá condenada a un marco institucional que no coincide con la realidad y a una apatía generalizada por los temas que no permiten el desarrollo del país, aunque se hable de ellos, no es tan atractivo hacerles frente. A continuación, se presentan una serie de gráficos de encuestas realizadas, unas enfocadas en la sociedad en general y otras en exclusivamente a los jóvenes de México.

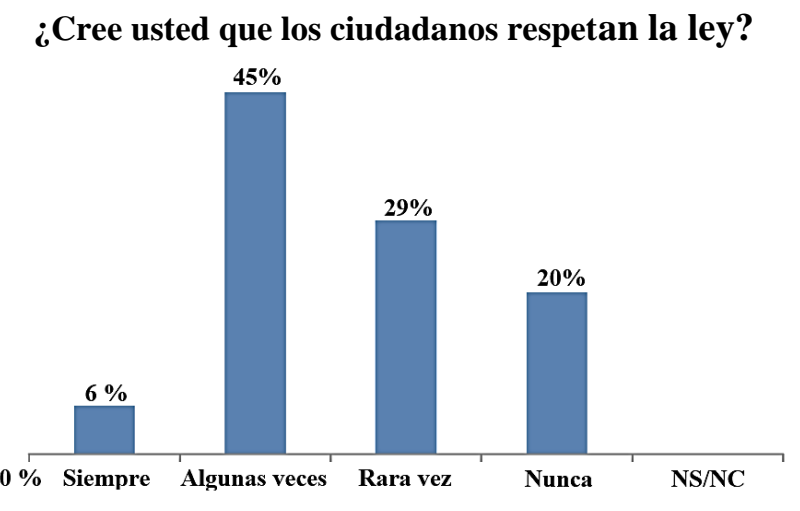

Gráfico 1 ¿Cree usted que los ciudadanos respetan la ley?

Fuente: elaboración propia a partir de la Encuesta Nacional de Valores del Banco Nacional de México; Revista Este País (2010, pág. 52)

En ésta otra gráfica se presenta otro dato preocupante, el 28 por ciento de los encuestados consideró necesario romper las leyes $\sin$ que otros se den cuenta para tener éxito. Aunque afortunadamente el 69 por ciento cree que hay que apegarse estrictamente a la ley, el 28 por ciento -equivalente a casi una tercera parte de los resultados- no lo considera así. En otras palabras uno de cada tres encuentra una relación entre éxito e ilegalidad. 


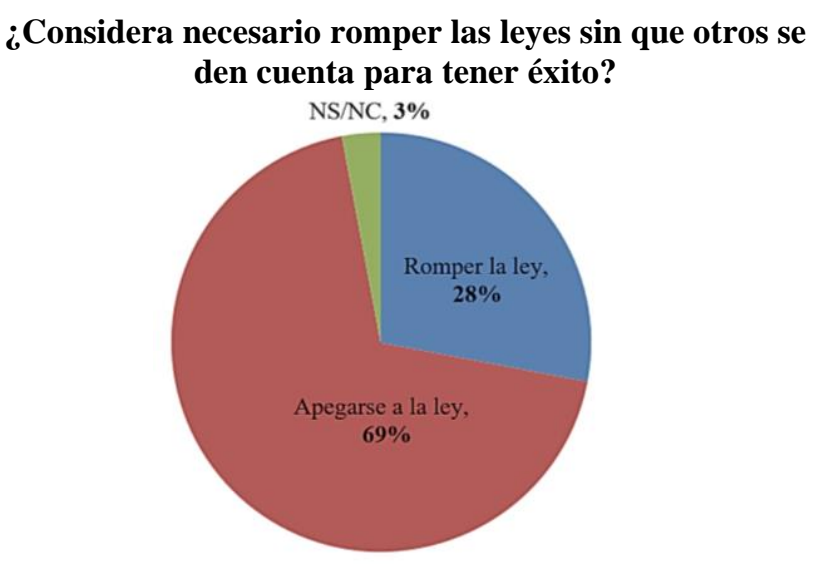

Gráfico 2 ¿Considera necesario romper las leyes sin que otros se den cuenta para tener éxito?

Fuente: elaboración propia a partir de la Encuesta Nacional de Valores del Banco Nacional de México; Revista Este País (2010, pág. 26)

Como se está evaluando el problema fiscal en función de los valores prevalecientes en los contribuyentes, probablemente ha aumentado la falta de valores como la tolerancia, compromiso, respeto, amor y la libertad. En lo relacionado a la credibilidad los gobernantes en cuanto a la toma correcta de decisiones solo el 4 por ciento considera que siempre los hacen así. En cuanto al rendimiento de cuentas a los ciudadanos también solo un 4 por ciento considera que lo hacen. Lo peor es que dos terceras partes consideran que los gobernantes "nunca" y "rara vez" respetan la ley.

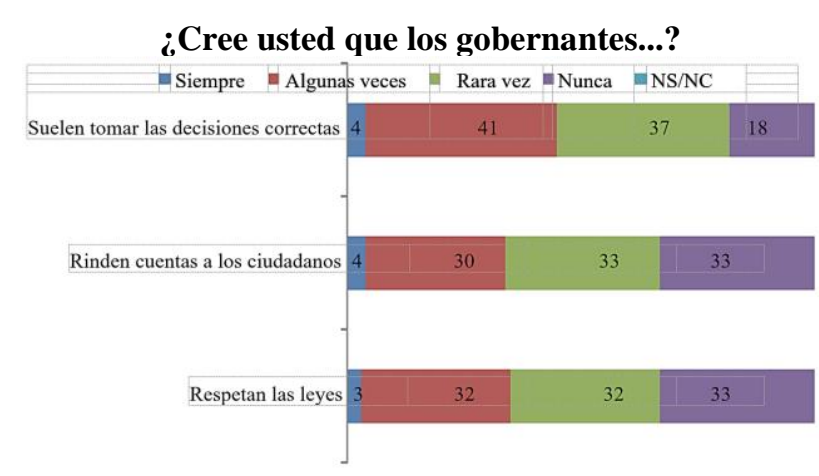

Gráfico 3 ¿Cree usted que los gobernantes...?

Fuente: Banco Elaboración propia a partir de la Encuesta Nacional de Valores del Banco Nacional de México; Revista Este País (2010, pág. s/n)

En la siguiente gráfica se muestran los resultados al preguntarle a la gente si prefería que bajara la carga tributaria aunque esto implicara disminuir la calidad de los servicios o si preferían mejores servicios aunque aumentara la carga de impuestos.

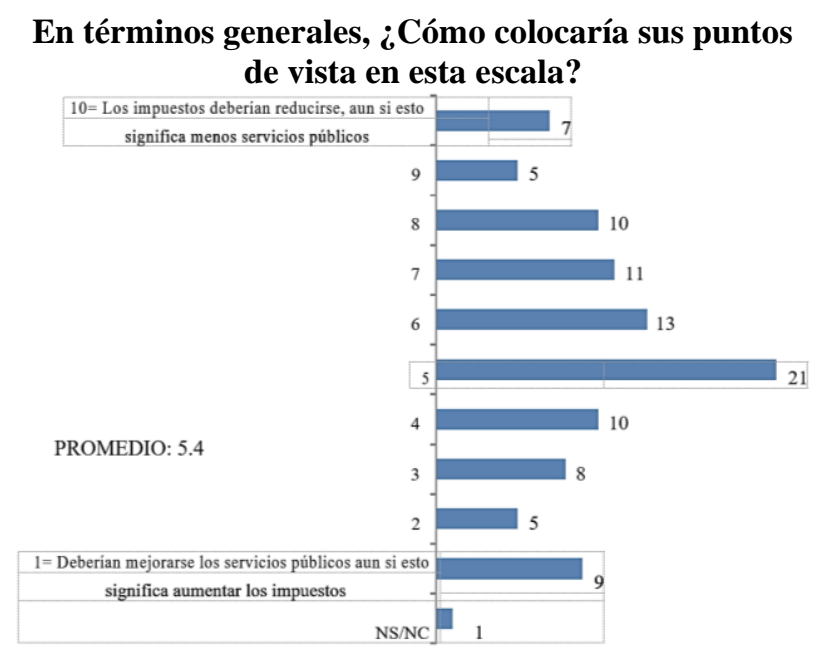

Gráfico 4 En términos generales, ¿Cómo colocaría sus puntos de vista en esta escala?

Fuente: Banco Elaboración propia a partir de la Encuesta Nacional de Valores del Banco Nacional de México; Revista Este País (2010, pág. s/n)

Los resultados indican casi una preferencia a que los impuestos no se modifiquen y se mantengan los niveles de servicios actuales aunque con un pequeño sesgo hacia la preferencia por la disminución de la carga tributaria.

\section{Metodología}

En esta sección del trabajo se describe cuál fue el procedimiento bajo el que se desarrolló la investigación que el lector tiene en sus manos. Para tal efecto, se mención el tipo de investigación de que se trata, cuáles fueron las variables consideradas en el estudio, bajo que enfoque se desarrolló la investigación y lo relativo al instrumento de investigación para la recolección de datos.

Dado que en esta investigación se abordó la problemática que enfrenta el sistema fiscal en México desde la perspectiva del sujeto obligado al pago de impuestos y de los servidores públicos obligados a realizar una correcta aplicación de los recursos públicos obtenidos, se decidió realizar una investigación no experimental, entendida como aquella en la que "no se construye ninguna situación, sino que se observan las ya existentes, no provocadas intencionalmente por el investigador" (Hernández Sampieri, Fernández Collado, \& Baptista Lucio, 2010, pág. 245). Se trató de un estudio de corte transeccional, ya que no se centró solamente en analizar la evolución de los valores a través de un periodo de tiempo determinado, sino que se recopiló información en un momento dado 
En esta investigación se exploró de manera general la relación existente entre los valores y la ética y los actores del sistema fiscal, por otro lado, con apoyo en los datos recabados con el instrumento de investigación, el trabajo también describió de manera objetiva el comportamiento ético que los sujetos encuestados manifestaron en sus respuestas, con relación al objeto de estudio de esta investigación. Lo anterior se complementó con una revisión documental a fin de fortalecer el argumento central de este trabajo, lo cual permitió tener mayores elementos para aceptar o rechazar los referidos supuestos.

Este trabajo tiene un enfoque mixto, es decir comparte características cuantitativas y cualitativas, apoyándose esta investigación en su mayor parte en datos recolectados a través de diversas encuestas, con el propósito de observar las tendencias y comportamientos de los resultados obtenidos. El estudio también fue cualitativo, porque "utiliza recolección de datos sin medición numérica para descubrir o afinar preguntas de investigación y puede o no probar hipótesis en su proceso de interpretación" (Sampieri, Collado, \& Lucio, 1991, pág. 10).

\section{Instrumento y técnicas de recolección de datos}

Como instrumentos y técnicas de investigación para la realización de este trabajo, se utilizaron las siguientes:

\section{Investigación documental.}

2. Investigación de campo Encuesta.

\section{Análisis y discusión de resultados}

En cuanto al tema de la percepción del mexicano moderno acerca los impuestos, se encontró que ellos perciben que: "Se paga demasiado en impuestos, se cree que el sistema beneficia a los que más tienen, se paga más en impuestos que lo que se recibe en servicios" (Casar, 2013, pág. s/n).
Como complemento a lo expuesto anteriormente, también se revisó lo concerniente a la evasión y la elusión, pero desde la óptica de los mexicanos y se encontró que las formas más comunes de evasión, tiene que ver con el nivel de conciencia al respecto, la percepción acerca de lo común o lo extendido del fraude fiscal en la población, el conocimiento de los medios de evasión, algunos elementos culturales y sociales presentes en el evasor y cómo percibe el contribuyente el balance entre el riesgo de evadir y el potencial castigo al que se expone.

Ante los resultados, es posible interpretar que a los encuestados les agravia más el monto que pagan de impuestos que el peculado y la mala fama que poseen los gobernantes. Esto representa que la mentalidad de las personas ha cambiado en torno a la corrupción.

Con respecto a la pregunta de: ¿Cuándo se debían de evadir impuestos?, dentro del mismo grupo de personas el 80.6 por ciento respondió que nunca, tal como se muestra en la gráfica, solo el 18 por ciento contempló escenarios donde se pueden evadir impuestos.

Se muestra que 94.4 por ciento de los encuestados consideran que hay mucha gente que no paga impuestos. Esto habla de la percepción de los respondientes en la cual, aun sin contar con datos estadísticos oficiales, la mayoría coincide en que la evasión de impuestos es una situación generalizada y ampliamente extendida en México.

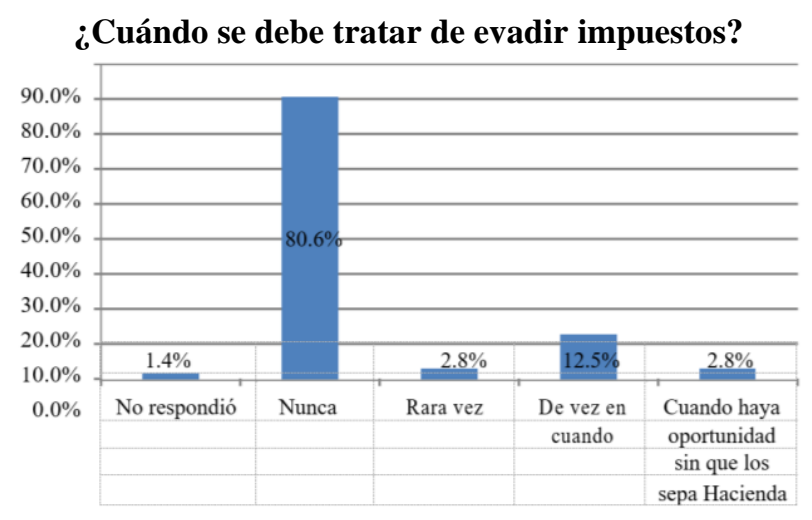

Gráfico 5 Cuando se debe tratar de evadir impuestos Fuente: Cárdenas Herrera, A., (2016) La Crisis de Ética como la posible causa raíz del problema fiscal nacional 
Como ya se ha mencionado con anterioridad, se ha dicho que la percepción de los contribuyentes es tan importante como los impuestos mismos, por lo tanto, el problema de la evasión fiscal es real y basándose en las percepciones de los encuestados y contestando esto con los reportes de Hacienda, respecto a la diminución de la evasión fiscal en el país resultan dos posturas diferentes que solo logran restarle credibilidad a los datos calculados por las autoridades oficiales.

En otro orden de ideas, la gráfica que sigue, muestra los resultados de la pregunta: ¿Evadir y eludir impuestos son prácticas que faltan a la moral? Al respecto, el 79.17 por ciento dijo que sí es una falta a la moral evadir y eludir. Esta proporción de encuestados el similar a las respuestas emitidas respecto a la pregunta: ¿Cuándo se debe tratar de evadir un impuesto? Esto significa que ante la generalidad, sigue siendo vista como una mala práctica la evasión de impuestos.

Los resultados de esta pregunta están alineados con los supuestos de investigación del presente trabajo, ya que es posible notar una influencia de la moralidad en la evasión y la elusión impuestos. Sin embargo, resulta muy temprano dar por hecho que este es el único factor que influye, pero existen elementos que permiten considerar que la moral y los principios éticos son factores determinantes en las personas al momento de dar cumplimiento de las obligaciones fiscales.

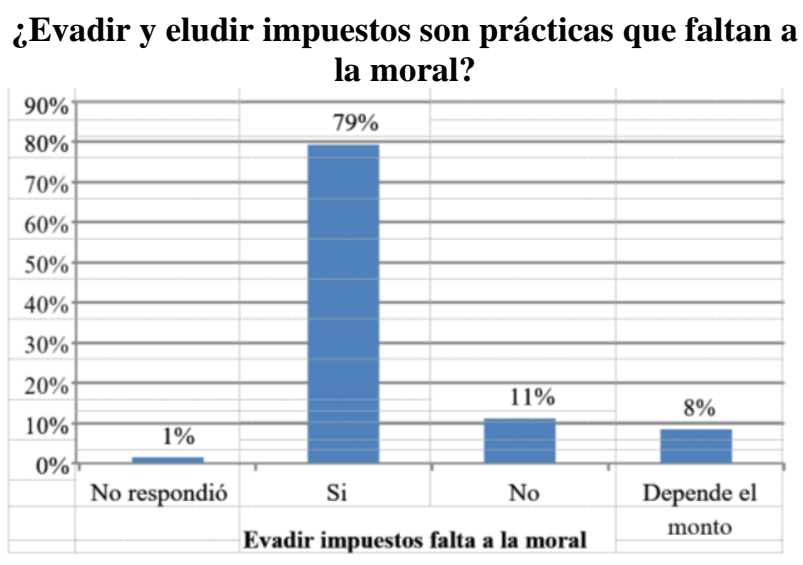

Gráfico 6 ¿Evadir y eludir impuestos son prácticas que faltan a la moral?

Fuente: Cárdenas Herrera, A., (2016) La Crisis de Ética como la posible causa raíz del problema fiscal nacional
Por lo que respecta a la forma de evasión consistente en la subdeclaración de ingresos, se les cuestionó a los encuestados si consideraban que declarar un "poquito" menos de los que ganan es evadir impuestos, esto con la intención de conocer si eran conscientes de esta forma de evasión. En este sentido, el 70.83 por ciento mostró ser consciente de esta forma de evasión. Un 15.28 por ciento lo sometería a una evaluación dependiendo el caso y casi un 14 por ciento cree que no es una forma de evadir. No obstante, lo anterior se encontró evidencia para señalar que existen personas que no catalogan la subdeclaración como una forma de evasión lo importante es que la mayoría si lo hace.

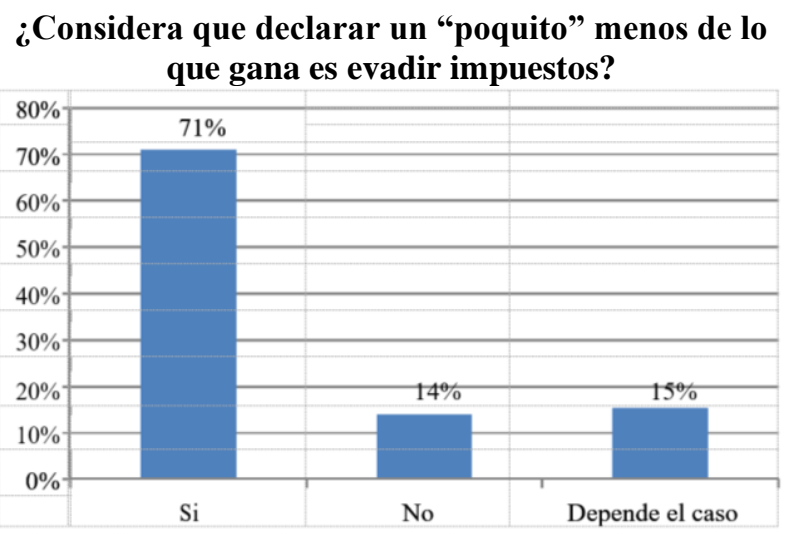

Gráfico 7 ¿Considera que declarar un "poquito" menos de lo que gana es evadir impuestos?

Fuente: Cárdenas Herrera, A., (2016) La Crisis de Ética como la posible causa raíz del problema fiscal nacional

Al pedirle a los encuestados que seleccionarán los valores más importantes que deben aplicarse para no evadir impuestos, se tuvieron respuestas muy homogéneas, siendo las siguientes en cuanto a contribuyentes cumplidos:

Honestidad, responsabilidad, integridad y conciencia social.

Bajo este mismo esquema, al pedirle a los encuestados que seleccionara tres valores de una lista, se les preguntó cuáles consideraban que eran los valores que a su juicio se han perdido y se resume lo siguiente:

El valor moral que a juicio de los encuestados, se ha perdido en mayor medida, fue el de la honestidad con una mención del 17.7 por ciento. En este caso, las respuestas en el grupo de edades de los 20 a los 49 años son muy homogéneas, en ambos casos rondando el 19 y el 16 por ciento. 
Por otro lado, el grupo de personas entre 50 y 59 años solo consideró en un 9.5 por ciento que se ha perdido este valor. Respecto al encuestado de 19 o menos años, éste mencionó también, que a su juicio este valor se ha perdido, cabe mencionar que este individuo en las tres preguntas de este tipo, hizo referencia a la honestidad, mencionando que se ha perdido, que es necesario para no evadir impuestos y que él la práctica; si bien es imposible llegar a un razonamiento concluyente, es importante notar que para este grupo de edad toma mucha relevancia este valor.

Es posible apreciar en la misma tabla, que el segundo lugar lo ocupó el valor del respeto, con un acumulado del 17.2 por ciento. En ese sentido cabe mencionar que este valor presentó casi la misma frecuencia que el valor de la honestidad. Para el rango de edad de 19 años o menos se presentó el mismo fenómeno descrito en el párrafo anterior. Así mismo, para el resto de los grupos la respuesta fue homogénea con porcentajes entre el 18.8 y el 11.1.

Se observa que la integridad fue el tercer valor con número de elecciones. El grupo que más consideró que este valor se ha perdido fue el de los 30 a los 39 años. Los grupos de 20 a 59 años seleccionaron también los valores de la conciencia social, la responsabilidad, la tolerancia y el valor de la familia como valores morales que se han perdido, con respuestas altamente homogéneas, las cuales varían entre el 5 y el 10 por ciento.

\section{Conclusiones y recomendaciones}

A partir de la información recabada a lo largo del presente trabajo, a continuación se presentan las conclusiones y recomendaciones obtenidas en esta investigación.

Considerando que el objetivo general del presente trabajo fue: Identificar la relación que existe entre la forma en que las personas viven los valores morales y la tendencia que muestran ante el cumplimiento de las obligaciones fiscales, a fin de sentar precedentes que permitan abordar el problema de los impuestos desde la óptica del comportamiento ético de los ciudadanos; es posible señalar que dicho objetivo se alcanzó tomando en consideración los siguientes aspectos: a) Se puede decir que sí existe una relación entre los valores morales y la tendencia que los informantes muestran ante el cumplimiento de las obligaciones fiscales; al menos así se observa este comportamiento en los resultados obtenidos en esta investigación.

b) Lo anterior así se puede señalar, en virtud de que se tuvo cuidado de que sólo se consideraran para la exposición de hallazgos, las preguntas formuladas en el instrumento de investigación que se aplicó y que no generaran sesgo en las respuestas emitidas.

Existe un aspecto que se mencionó a lo largo de la investigación, el cual influye en gran medida en la voluntad de los contribuyentes a pagar estrictamente los impuestos o a estar buscando la forma de pagar menos o en su defecto, no pagar.

Se dijo que la percepción respecto a la justicia de los impuestos mejora o empeora la disposición de los ciudadanos frente a las obligaciones fiscales. Es importante considerar que el término referido a justicia se aborda desde dos aspectos:

Derivado de las complicaciones ya planteadas, surgen preguntas como:

¿Es mucho o poco el impuesto respecto a otros países? ¿Es mucho o poco el impuesto respecto a la proporción del ingreso? ¿Es mucho o poco el impuesto respecto a una calle de asfalto o de concreto hidráulico? ¿Es mucho o poco el impuesto respecto a la seguridad de una u otra colonia? ¿Debería pagar más impuesto la gente que utiliza los servicios públicos como centros de salud y escuelas públicas, respecto de aquellos ciudadanos que no los utilizan? 
Derivado de la imposibilidad de contestar estas interrogantes y muchas otras, que permitirían especificar los criterios para satisfacer las demandas de aquellos que perciben que los impuestos son injustos, la única recomendación que se le puede hacer a la autoridad, es que ésta debería sería hacer campañas de difusión masiva con el mensaje, "tus impuestos permitieron esta obra de infraestructura que beneficia a tantas personas", "tus impuestos permitieron equipar a la policía y pagar mejores salarios a los elementos que detuvieron a tal delincuente de alta peligrosidad", "con la ayuda de tus impuestos, tantas mujeres recibieron un trato digno $\mathrm{y}$ apoyo médico en su labor de parto", "tus impuestos permitieron la inversión de tal cantidad de dinero en mejoras para la ciudad, esto ayudó a la atracción de tales fuentes de empleo para ti y tu familia".

La efectividad y veracidad de estos mensajes depende de la imparcialidad con la que se emitan; es decir, las veces que han existido mensajes similares no tienen el enfoque que ayuda a revertir esta tendencia en el pensamiento de los ciudadanos, ya que en la mayoría de las ocasiones, parecen mensajes con tendencia partidista o para justificar los actos del gobierno. La clave sería hacer sentir a la gente que ellos aportan, contribuyen y son parte de que las cosas cambien y mejoren con la ayuda del dinero que ellos aportan al gobierno. Es convertir al ciudadano en un socio de su colonia, ciudad y país donde lo que hace es inyectarle capital periódicamente para el desarrollo de dicha "empresa". La corrupción y peculado cuando salen a la luz pública, contribuye a la sensación de injusticia en los impuestos a pagar. En este sentido, existen dos partes a considerar:

- La primera consiste en que el mal proceder de algunos funcionarios públicos no debe ser justificación para que las personas no cumplan con el pago de sus impuestos; es cierto, a nadie le agrada pagar impuestos y ver cómo alguien se enriquece con el esfuerzo de otros, peor aún, cuando impunemente no es juzgado ni castigado por ello. Esta actitud solo convierte en esclavos de las demás a las personas que se valen de los actos de otros para justificar los propios, ya que pierden el control de sí mismos, pierden su libertad.
- La segunda parte a considerar cuando se habla de funcionarios corruptos, es el avance que se está dando en la lucha contra esta práctica que lastima al país.

Por lo que se refiere al objetivo de Identificar los valores presentes en los contribuyentes cumplidos y en los evasores, al respecto, se identificaron los siguientes: Responsabilidad, respeto y honestidad.

Cabe señalar que estos valores fueron mencionados indistintamente por los contribuyentes cumplidos y por los evasores; sin embargo, es importante resaltar que la diferencia principal radica en que los contribuyentes cumplidos, catalogados a través de diferentes preguntas de verificación, fueron congruentes con los valores que señalaron practicar.

En cuanto al segundo objetivo específico, que se enunció de la siguiente manera: Revisar las variaciones que se presentan en los valores que viven los contribuyentes según la generación a la que pertenezcan, se encontró que en este caso sí existen variaciones, se puede apreciar que los grupos generacionales de los informantes cuya edad estaba comprendida de los 19 años $o$ menos hasta los 39 años en la fecha en que se aplicó el instrumento de investigación, coinciden entre ellos respecto a los valores que mencionaron practicar; sin embargo, de los 40 años en adelante, el valor de la honestidad no aparece en la terna de los valores más representativos, en cambio, coincide que dichos informantes mencionan el valor de la familia y la tolerancia. Lo anterior demuestra que existe una variación en los valores que presentan las diferentes generaciones.

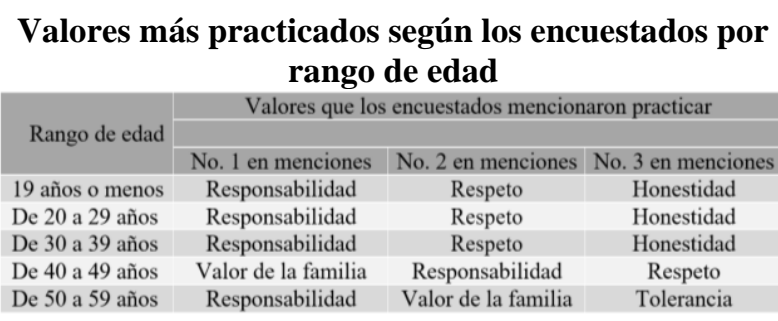

Tabla 1 Valores más practicados según los encuestados por rango de edad

Fuente: Cárdenas Herrera, A., (2016) La Crisis de Ética como la posible causa raíz del problema fiscal nacional 
Algunos aspectos saltan a la vista, y sería muy interesante que trabajos futuros corroboren éstos. Dichos aspectos no fueron detallados en el presente trabajo porque el enfoque principal exigía mirar siempre a la problemática fiscal y no el comportamiento social como eje fundamental de esta investigación. En cuanto al objetivo específico, consistente en: Verificar el nivel de conocimientos básicos en materia fiscal por parte de los contribuyentes, se logró identificar que el nivel de conocimientos en materia fiscal, aún no es suficiente para poder descartar esta variable como una posibilidad de incumplimiento fiscal.

Si bien es cierto que el desconocimiento de la ley no exime a ninguna persona respecto a su cumplimiento, en México aún resulta un factor que influye en la razón por la cual los ciudadanos no enteren el tributo que les corresponde de acuerdo a sus actividades económicas. Esta situación se acentúa en la media que los contribuyentes cuenten con niveles académicos menores. Es importante destacar que mientras esta variable no se controle en la ecuación del incumplimiento fiscal, no será posible asegurar que los evasores actúan en todas las ocasiones con dolo contra la autoridad fiscal.

Como parte de las mismas conclusiones de este trabajo, en lo subsecuente se presentan los resultados correspondientes a los cuatro supuestos planteados en el primer capítulo de esta investigación:

Por lo que hace al supuesto, Existe una fuerte influencia de los valores y la ética en el comportamiento de los evasores de impuestos, este se cumplió parcialmente. De acuerdo a los resultados arrojados por la encuesta, sí existe una influencia de los valores de cada persona en su comportamiento fiscal; sin embargo, no existen elementos suficientes pare determinar que esta influencia es "fuerte".

No obstante lo anterior, existe otro grupo de contribuyentes que cumplen con sus deberes fiscales; sin embargo, no lo hacen por convicción, si no por miedo a la autoridad fiscal y al castigo que pudiera implicar el omitir el pago de sus impuestos. En este grupo de contribuyente no hay nada admirable, ya que se trata de contribuyentes que hacen gala de una actitud pueril en la que su único motivo para obrar bien es no ser castigados.
Este comportamiento demerita en todo sentido a la virtud humana, ya que expone el hecho de que nunca existió, ya que como se ha comentado con anterioridad en este documento, la ética reside en que el hombre actúe en el bienestar propio y de su comunidad, independientemente de la existencia de factores extrínsecos como el miedo al castigo por evasión, que en este caso se plantea.

En cuanto al segundo supuesto: Los medios masivos de comunicación influyen fuertemente en la deformación de los valores éticos, se puede decir que este se cumple satisfactoriamente. A través de la encuesta realizada, se confirmó el escenario teórico documentado a lo largo de la presente investigación, todo converge en que, al menos en México, se puede decir que la televisión es un elemento que ha influenciado negativamente sobre la aplicación de los valores y los parámetros de permisividad con la que operan las nuevas generaciones. Es evidente que el internet en la actualidad es un medio de comunicación con el que las nuevas generaciones están fuertemente vinculadas; por lo que se puede señalar que el internet también contribuye en gran parte en la tendencia creciente de la deformación de los valores.

Respecto al tercer supuesto, el cual se enuncia de la siguiente manera: La práctica de los valores y la ética es esencial para el sano funcionamiento del sistema fiscal mexicano, se puede decir que se cumple parcialmente. Este supuesto se halla estrechamente vinculado al primero, por lo tanto también se cumple parcialmente en el siguiente sentido:

La ética es esencial para el sano funcionamiento de todo, en tal caso, esto aplica para el sistema fiscal en México, sin embargo, no es lo único. Hace falta también un acercamiento a las necesidades y posibilidades de los contribuyentes, así como informar, transparentar y fomentar la cooperación entre la autoridad y los ciudadanos, simplificar el sistema, cambiar la relación terrorista existente entre la autoridad y el contribuyente y otras acciones.

A través de esta información se detectó que cada grupo generacional encuestado considera en buena medida, que las generaciones más jóvenes viven y aplican poco los valores. 
Aunque la información recolectada en la encuesta no permite describir exactamente qué sucede respecto a los valores en cada generación, si se puede decir que cada una de ellas evalúa negativamente los valores presentes en las generaciones que le suceden, lo cual es una evidencia de la diferencia que existe en los valores que cada generación aplica.

\section{Referencias}

Argudín, Y., \& Luna, M. (2001). Desarrollo del pensamiento crítico. México: Universidad Iberoamericana-Campus Santa Fe / Editores Plaza y Valdés.

Aristegui noticias. (10 de diciembre de 2012). Aristegui noticias. Recuperado el 12 de octubre de 2014, de http://aristeguinoticias.com/1012/mexico/cepalcritica-a-mexico-por-su-baja-recaudacion-deimpuestos/

Banco Nacional de México; Revista Este País. (2010). Encuesta Nacional de Valores. Recuperado el 18 de enero de 2014, de Encuesta Nacional de Valores: http://www.observatoriopropaz.org.mx/Docum entos/formacion/2.pdf

Canonici, T. (2013). Millennials: una nueva generación. Recuperado el 8 de julio de 2014 de Leanstart.es: http://www.leanstart.es/millennials-una-nuevageneracion/

Cantalá, D., Sempere, J., \& Sobarzo, H. (2005). Evasión fiscal en el impuesto sobre la renta de personas físcas. México: Centro de Estudios Económicos de El Colegio de México.

Casar, M. A. (1 de noviembre de 2013). Los mexicanos contra los impuestos. Recuperado el 16 de agosto de 2014, de Revista Nexos: http://www.nexos.com.mx/?p=15579

Casar, M. A., \& Buendía, J. (1 de enero de 2001). El mexicano ante los impuestos. Recuperado el 12 de agosto de 2014, de Revista Nexos: http://www.nexos.com.mx/?p=9880

Centro de Investigación para el Desarrollo, A.C. (2011). Encuesta de valores México.
Endo Martínez, A. M., Mendoza Montenegro, V., \& Zorrilla Mateos, F. M. (2006). Medición de la Evasión Fiscal en México. México: Centro de Economía Aplicada y Políticas Públicas.

Real Academia Española. (3 de agosto de 2014). Crédito. Recuperado el 3 de agosto de 2014, de Real Academia Española: http://lema.rae.es/drae/srv/search?id=zD67MzL bUDXX20Qi9Fnw

Reyes Heroles, F. (1 de octubre de 2002). La revolución de los valores. Recuperado el 15 de agosto de 2014, de Revista Nexos: http://www.nexos.com.mx/?p=10610

Wolf del Valle, N. J. (1 de abril de 2006). Evasión fiscal. Un estudio econométrico. Nuevo Consultorio Fiscal. 